\title{
Diagnostic efficacy and safety of transperineal prostate targeted and systematic biopsy: The preliminary experience of first 100 cases
}

\author{
Shahbaz Mehmood, Khalid Ibraheem Alothman, Abdulaziz Alwuhaibi, Samia Mohamed Alhashim \\ King Faisal Specialist Hospital \& Research Center, Riyadh, KSA.
}

\begin{abstract}
Summary Background: Post-biopsy urosepsis is a major concern for patient morbidity and cost. Trasperineal biopsy is reported to have less complications and higher detection rate of clinically significant prostate cancer (csPCa).

Objectives: To determine the diagnostic efficacy and safety of transperineal prostate biopsy in patients with elevated prostatic specific antigen (PSA).

Material and methods: A prospective study included men with elevated PSA > $3 \mathrm{ng} / \mathrm{ml}$ and previous negative biopsy from January 2018 to April 2019. All patients had multiparametric magnetic resonance imaging (mpMRI) and suspicious lesions reported as Prostate Imaging Reporting and Data System (PIRADS) score version 2. Average twelve systematic and two targeted cores were biopsied under general anaesthesia. Patients received single dose of antibiotic prebiopsy.

Results: 100 Consecutive patients having median age 64.0 years and median PSA of $6.1 \mathrm{ng} / \mathrm{ml}$ were included for $\mathrm{mpMRI-US}$ fusion transperineal biopsies. Cancer detection rate was $45 \%$ (targeted $38 \%$ and systematic 22\%) and csPCa were detected in $75.55 \%$ (targeted $86.84 \%$ and systematic $59.09 \%$ ). MRI-US fusion targeted biopsies detected $63.88 \%$ csPCa in PIRADS 5, $33.33 \%$ in PIRADS 4 and $5.88 \%$ in PIRADS 3 lesions. PSA $>10$ $(p=0.012)$, PSA density $>0.15(p=0.0002)$, and PIRADS 5 (0.0001) were significantly associated with PCa. Factors like Age (0.0001), initial PSA (0.022) and PSA density (0.006) were significant on univariate analysis while age (0.0001) was significant on multivariate analysis. There was no case of urinary tract infection.

Conclusions: Transperineal prostate biopsy is safe and effective in diagnosing csPCa. There is no risk of sepsis and major complications.
\end{abstract}

KEY WORDS: Multiparametric MRI; Transperineal; Urosepsis; Targeted biopsy; Systematic biopsy.

Submitted 19 October 2020; Accepted 28 December 2020

\section{INTRODUCTION}

Prostate cancer (PCa) is the most common solid cancer in men and third leading cause of death in developed world (1). Traditionally, PCa is diagnosed with transrectal ultrasound guided biopsy (TRUSBx) in a patient with high Prostatic Specific Antigen (PSA) and or abnormal digital rectal examination. However, most studies $(2,3)$ and
American Urological Association (AUA) white paper (4) determined that transrectal ultrasound guided biopsy (TRUSBx) is associated with 5 to $7 \%$ of risk of infection and 2 to $4 \%$ of hospital admission despite giving prophylactic antibiotics.

For the last decade, transperineal TRUS guided prostate biopsy (TTPB) is gaining popularity in the diagnosis of prostate cancer (5). As there is no intrusion of gastrointestinal or urogenital tract, TTPB is relatively considered a clean procedure. There is an ample published evidence that TTPB carried a very low risk of infection and hospital re-admission rate as compared to TRUSBx (6-8). TTPB is mostly performed under general anaesthesia but some centres have even reported under local anaesthesia (9). Similarly, there is an increasing evidence that multiparametric magnetic resonance imaging (mpMRI) of prostate can helps diagnosing high risk prostate cancer and decreases number of unnecessary biopsies (30). In order to increase cancer detection rate, concept of mpMRI detected suspicious areas were targeted with transrectal ultrasound guidance biopsy (10). Transperineal mpMRIUS fusion biopsy has advantage of detecting more cancers in anterior prostate as compare to transrectal mpMRI-US fusion biopsy (11). As for as targeted or systematic biopsy is concerned, although mpMRI has highest detection rate of clinically significant prostate cancer, combination of targeted and systematic mpMRI-US fusion transperineal biopsy are still required $(12,13)$.

In spite of highest detection rate, mpMRI may still miss $8-24 \%$ of clinically significant prostate cancer (csPCa) if we compare total foci detected on prostatectomy specimen with mpMRI detected lesions $(14,15)$.

In this prospective study we will report our experience of first hundred patients with mpMRI-US fusion transperineal prostate biopsy under general anaesthesia. Primary aim of this study is to detect infectious complications and cancer detection rate. The ultimate goal is to reduce infectious complications, increase detection rate of csPCa and reduce unnecessary biopsies.

\section{Materials And methods}

This prospective study was approved by office of research assistant (ORA) an institutional review board of king

No conflict of interest declared. 
Faisal Specialist Hospital \& Research Centre Riyadh. Informed written consent was taken from every patient before MRI-US fusion transperineal biopsy. From January 2018, we converted to transperineal MRI-US fusion prostate biopsy from TRUSBx in all patient with elevated PSA and suspicious mpMRI. Between January 2018 and July 2019, an hundred consecutive patients were recruited prospectively for transperineal prostate biopsy. All patients who had high PSA $>3.0 \mathrm{ng} / \mathrm{ml}$ with reference to age and previous negative conventional biopsy were included and subjected to pre biopsy multiparametric MRI (mpMRI) by our dedicated uro-radiologist. Patients in whom MRI was contraindicated or refused by patients either for mpMRI or for transperineal biopsy were excluded from study. Patient in whom normal mpMRI $[<$ Prostate imaging reporting and data system (PIRADS) Score 3] and high PSA were also included for systematic biopsy.

\section{mpMRI and biopsy protocol}

All patients underwent pre biopsy mpMRI of prostate. MRI images were reviewed by our dedicated Uro-radiologist and reported with PIRADS version 2 (16). These mpMRI images were stored in hospital computer ICIS network and imported for fusion with real-time TRUS via localized network system. MRI/US fusion-guided biopsy was performed with the BioJet fusion system and software (DEK Technologies, Barum, Germany). A minimum of one and preferably 2-4 cores were taken from each target lesion. A systematic 12 core transperineal biopsies were performed in every patient after targeted biopsy.

All patients received only single one-gram Cephazolin intravenously at the time of induction of general anaesthesia and procedure was performed under dorsal lithotomy position. Patients were only given analgesics in the form paracetamol and no oral postbiopsy antibiotics. Biopsies were done by a single urologist who had an experience in transperineal mpMRI-US fusion prostate biopsy.

\section{Outcome}

We investigated the clinical safety in term of complications like urinary tract infection, sepsis, hospital admission rate, urinary retention, pain and perineal hematoma. Moreover, overall cancer detection and clinically significant prostate cancer detection rate in targeted and systemic biopsies were analysed. A clinically significant prostate cancer was defined as Gleason score of $\geq 7$, Gleason score $\geq 2$ positive cores, and bilateral cancer on prostate biopsy (17).

\section{Statistical analysis}

Analysis of patient demographics along with clinical and pathologic variables using descriptive statistics was performed. Fisher's exact test and chi-square test were used for statistical analysis for categorical variables and student's t-test for continuous variable and are specified as percentage. Statistical analysis was done using the SAS software package, version 9.4 (Statistical Analysis System, SAS Institute Inc., Cary, NC, USA). Univariate logistic regression analysis was used to determine the association between baseline patient characteristics like age, body mass index (BMI), PSA, PSA density and detection of PCa.
Multivariable regression analysis was used to investigate the association between PCa detection and variables which were significant in univariate regression model. A p-value of $<0.05$ was considered statistically significant.

\section{Results}

Between January 2018 and June 2019, 100 consecutive patients with clinically and biochemically suspected for PCa were recruited for transperineal mpMRI-US fusion prostate biopsy. Median age of patients was 64.0 (60.072.0) and median initial PSA and PSA density were 6.1 $(4.8-11.5) \mathrm{ng} / \mathrm{ml}$ and $0.12(0.10-0.27) \mathrm{ng} / \mathrm{ml}^{2}$ respectively. Mean time from mpMRI to transperineal targeted biopsy was $4.1 \pm 3.6$ months. Average two targeted and 12 systematic biopsies were taken in every patient.

A total of 45 patients (45.0\%) were diagnosed as prostate cancer. Among these, 22 patients were diagnosed as cancer on systematic biopsy while 38 patients were found to have cancer on targeted biopsy. Similarly, detection rates of csPCa were $13 / 22$ patients (59.09\%) in systematic biopsy while $33 / 38$ patients (86.84\%) were in targeted arm and $34 / 45$ patients $(75.55 \%)$ in combination. Systematic biopsy detected more (10\%) insignificant prostate cancer than targeted biopsy (5\%). Among 45 patients, 16 patients had a prostate cancer diagnosed on both systematic and targeted biopsy and 3 of them had discordant Gleason score between systematic and targeted biopsies and these patients were upgraded to targeted biopsy cores because of Gleason score. Similarly, 22 (22\%) patients were exclusively diagnosed on targeted biopsy while systematic biopsy diagnosed exclusively $7(7.0 \%)$ patients.

\section{Prostate cancer detection and mpMRI PIRADS score} Among mpMRI and its reporting on the basis of PIRADS version 2, 20 patients (20.0\%) without suspicious lesion on mpMRI (PIRADS < 3) and high PSA, systematic biopsies revealed none of the patient with prostate cancer; 17 (17.0\%) patients had PIRADS 3, targeted biopsies detected only 2 patients of whom one cancer of clinically significant and one with insignificant PCa while systematic biopsies confirmed 3 cancer patients, of whom only one patient was having csPCa; 27 patients $(27.0 \%)$ had PIRADS 4 lesions, targeted biopsies diagnosed 11 cancer patients (40.74\%), of whom 3 had GS 6, 6 GS 3+4, and 2 GS 4+3 while systematic biopsies detected 6 patients (18.51\%), of whom 4 had GS 6 and 2 GS 3+4; lastly 36 patients (36.0\%) were reported as PI-RADS 5, targeted biopsies detected 25 patients (69.44\%) cancer, of whom 2 patients had GS 6, 6 GS 3+4, 3 GS 4+3, 3 GS 8, 9 GS 9 , and 2 GS 10 while systematic biopsies diagnosed cancer in 14 patients (38.88\%), of whom 4 patients had GS 6, 3 GS 3+4, one GS 4+3, 2 GS 8, 2 GS 9, and 2 with GS 10. A total of 8 patients had prior negative prostate biopsy with conventional TRUSBx, three (37.5\%) were diagnosed as cancer on transperineal mpMRI-US fusion biopsy. All 3 patients were having csPCa, found in anterior prostate and none had cancer diagnosed on systematic biopsy as shown in Table 1 .

We categorised PSA into $<4,4-10$ and $>10 \mathrm{ng} / \mathrm{ml}$ and found that higher the PSA, more patients with PCa were diagnosed $(p=0.0127)$. Similarly, PSA density $>0.15$ 
Table 1.

PI-RADS Score and PCa detection rate.

\begin{tabular}{|l|c|ccc|c|ccc|l}
\hline Variables & Total & \multicolumn{2}{|c|}{$\begin{array}{c}\text { Systematic biopsy } \\
\text { Benign cisPCa cSPCa }\end{array}$} & p-value & \multicolumn{2}{|c|}{$\begin{array}{c}\text { Targeted biopsy } \\
\text { Benign cisPCa }\end{array}$} & csPCa & -value \\
\hline PIRADS <3 & 20 & 20 & 0 & 0 & & 20 & 0 & 0 & \\
PIRADS 3 & 17 & 14 & 02 & 01 & 0.0214 & 15 & 01 & 01 & $<0.0001$ \\
PIRADS 4 & 27 & 22 & 03 & 02 & & 16 & 02 & 09 & \\
PIRADS 5 & 36 & 22 & 04 & 10 & & 11 & 02 & 23 & \\
Prior negative biopsy & 08 & 08 & 0 & 0 & & 05 & 0 & 03 & \\
\hline
\end{tabular}

PI-RADS: Prostate imaging reporting and data system; PCa: Prostate cancer,

cisPCa: Clinical insignificant prostate cancer; csPCa: Clinically siǵnificant prostate cancery.

Table 2.

PSA, PSA density and overall malignancy rate.

\begin{tabular}{|c|c|c|c|c|}
\hline Variables & Total & Benign & Malignant & p-value \\
\hline \multicolumn{5}{|l|}{ PSA } \\
\hline$<4$ & 11 & 9 & 2 & \multirow{3}{*}{0.0127} \\
\hline $4-10$ & 60 & 36 & 24 & \\
\hline$>10$ & 29 & 10 & 19 & \\
\hline \multicolumn{5}{|l|}{ PSA density } \\
\hline$<=0.15$ & 66 & 45 & 21 & \multirow[t]{2}{*}{0.0002} \\
\hline$>0.15$ & 34 & 10 & 24 & \\
\hline
\end{tabular}

Table 3.

Univariate and multivariate analysis of variables for overall detection of prostate cancer.

\begin{tabular}{|l|ccc|ccc|}
\hline \multirow{2}{*}{ Variables } & \multicolumn{3}{|c|}{ Univariate analysis } & \multicolumn{3}{c|}{ Multivariate analysis } \\
& OR & $95 \% \mathrm{Cl}$ & $\mathrm{p} \mathrm{value}$ & $\mathrm{OR}$ & $95 \% \mathrm{Cl}$ & $\mathrm{P}$ value \\
\hline Age & 1.181 & $1.097-1.272$ & $<0.0001$ & 1.193 & $1.098-1.295$ & 0.0001 \\
\hline BMl & 1.018 & $0.921-1.124$ & 0.7318 & & & \\
\hline Initial PSA & 1.071 & $1.010-1.136$ & 0.0228 & 0.947 & $0.868-1.033$ & 0.5127 \\
\hline Prostate vol. & 0.996 & $0.979-1.014$ & 0.6934 & & & \\
\hline PSA density & 109.41 & $3.761->999.99$ & 0.0063 & 500.29 & $0.695->999.99$ & 0.0641 \\
\hline PSA: Prostate specific antigen; 0D: Odd ratio; BMl: Body mass index; Vol.: Volume; Cl: Confidence interval. \\
\hline
\end{tabular}

was significantly associated with PCa as in Table 2. In order to identify factors associated with prostate cancer, univariate logistic regression analysis was done. Factors like age, initial PSA and PSA density were significant associated with prostate cancer. When these significant factors were analysed by multivariate regression analysis, only age was the significant factor for PCa as in Table 3.

\section{Transperineal biopsy and complications}

In our series of 100 cases of mpMRI-US fusion TPBx, few minor and no major complication was reported following TPBx. Five patients (5.0\%) presented with urinary retention in emergency room; 3 of them were already having lower urinary tract symptoms pre biopsy and taking alpha blocker. These patients had significantly greater mean prostatic volume $\left(70.2 \mathrm{~cm}^{3}\right)$ than patient who did not develop $\left(49.8 \mathrm{~cm}^{3}\right)(\mathrm{p}=0.024)$. Similarly, more systematic biopsy cores (15.5) than patients who did not develop retention (12.0) ( $p=0.012)$. Urine cultures were negative in these patients. All had temporary catheterization, of whom 4 had successful trial without catheter and one ended up transurethral resection of prostate. Almost all patient had mild temporary hematuria which settled down in 48-72 hours while complaint of hematospermia remained for 2-3-month post biopsy. Temporary skin bruising was noted in almost all patients. All patients received single intravenous dose of antibiotics at induction of anaesthesia. None of patient received postoperative oral antibiotics. Most importantly, there was not a single case of urosepsis who needed hospital admission.

\section{Discussion}

Traditionally, PSA has been used for prostate cancer screening and TRUS guided 10-12 cores systematic biopsy was considered a diagnostic method for prostate cancer for more than two decades. However, this diagnostic method no doubt detects prostate cancer but also detect insignificant prostate cancer which leads to over diagnosis. Therefore, systematic biopsies have low sensitivity and tend to diagnose more insignificant PCa (18).

In order to improve detection of clinically significant PCa and reduce rate of negative biopsies, mpMRI targeted biopsies has been reported a high sensitivity and specificity in many studies $(19,20)$. PIRADS version 2 categories were assigned prospectively to all lesions in our series. This system was established in 2012 and updated in 2014 as PIRADS version 2. PIRADS v2 dictates that scores 3-5 lesions should be subjected to MRI image guided targeted biopsies and found to have excellent detection rate for $\mathrm{PCa}$ and $\operatorname{csPCa}(21,22)$. In our study, we found highest overall PCa (40.74\% \& 69.44\%) and csPCa (33.33\% \& 63.88\%) detection rate for PIRADS 4 $\& 5$ respectively. Twenty patients with PIRADS score $<3$ with high PSA > 4 underwent systematic biopsies, all turned out to be negative.

Similarly, in our series with relatively low rate of PCa detection, mpMRI-US fusion biopsies diagnosed cancer in $45.0 \%$ of the patients; $75.55 \%$ of these cancers were clinically significant, and targeted biopsies alone detected 33/80 (41.25\%) of csPCa. Moreover, MRI-US fusion targeted biopsies missed seven cases, including two having csPCa which were diagnosed on systematic biopsies. This stresses the essentiality of systematic cores during prostate biopsy.

There are inconsistent results in detection rates of targeted and systematic biopsies. One of the prospective study of MRI/US fusion targeted versus concurrent systematic transperinel biopsy showed that detection rate of csPCa was higher in systematic biopsy arm compared to targeted biopsy $57.1 \%$ vs $48.0 \%$, p = 0.088 (23).

In contrast Valerio et al. performed a systemic review on 15 studies and found a consistent results that MRI-US fusion targeted biopsies diagnosed more csPCa (median: $33.3 \%$ vs $23.6 \%$ ) compared to conventional random biopsy technique and even MRI-US fusion targeted biopsies detect csPCa (median: 9.1\%; range: 5-16.2\%) that would have been missed by conventional biopsy (24). In our series, we had only eight cases with high PSA and prior negative conventional TRUS guided biopsies.

Of whom 3 cases diagnosed with csPCa on MRI-US fusion targeted biopsy and all were anteriorly located suggesting the necessity of MRI-US fusion targeted transperineal 
approach for prostate biopsy in these anterior located lesions. Similarly, D'Agostino et al. (25) found that MRI-US fusion targeted biopsy is safe and highly accurate for diagnosing csPCa especially in patients with prior negative TRUS guided prostate biopsy in their 155 patients.

The primary objective of this study was to determine diagnostic efficacy and safety of transperineal mpMRI-US fusion prostate biopsy. From safety perspective, we have not found a single case of urosepsis in our series. Five patients developed urinary retention, received temporary catheterization and one of them ended up TURP as he was on alpha blocker and having preoperative lower urinary tract symptoms. Similarly, Vyas et al. (26) found a similar result in relatively large series. There was not a single case of urosepsis and $1.7 \%$ of cases developed temporary urinary retention post transperineal biopsies. It is well recognised that infectious complications and after TRUSBx is steadily increasing worldwide. Multidrug resistant organisms are also increasing due to repeated use of antibiotics $(27,28)$ Lack of infectious complications makes transperineal prostate biopsy alternative to transrectal biopsy. Transperineal biopsy should be considered a clean procedure as neither gastrointestinal nor urinary tract is traversed. Similarly, Pepe et al. (31) presented the morbidity and clinical complications of transperinal biopsy in 3000 patients and found that complications are directly related with number of biopsy cores taken

Our study is strengthened because of its prospective nature, single institution and single surgeon performed the biopsy. There are also limitations of our study.

First, there was no control arm like patients with systematic TRUS guided non fusion biopsies. Although mpMRI reporting were done by our dedicated uro-radiology team but mpMRI reporting and practical use of MRI for prostate biopsies obviously needs a learning curve (29). Moreover, this study is limited due to nonvalidation of biopsy results with histopathological finding of whole gland prostatectomy specimen. Although we have got csPCa and index lesions correspond to targeted biopsy results, it cannot be said with certainty that all csPCa has been detected without histological analysis of whole gland specimens.

Next, the number of patients in our study was relatively low to accurately determine the cancer detection rate. In addition, transperineal prostate biopsy is safer than transrectal biopsy with regards to lower risk of sepsis and hospital re admission. Although some centres have published transperieal biopsy under local anaesthesia successfully but this is a disadvantage in our series by using general anaesthesia.

\section{Conclusions}

Transperineal mpMRI-US fusion prostate biopsy is highly accurate and safe in diagnosing clinically significant prostate cancer. There is no risk sepsis or major complications.

\section{REFERENCES}

1. Jemal A, Bray F, Center MM, et al. Global cancer statistics. CA Cancer J Clin. 2011; 61:69-90.
2. Loeb S, Carter HB, Berndt SI, et al. Complications after prostate biopsy: data from SEER-Medicare. J Urol. 2011; 186:1830.

3. Bennett HY, Roberts MJ, Doi SA, et al. The global burden of major infectious complications following prostate biopsy. Epidemiol Infect. 2016; 144:1784-91.

4. Liss MA, Ehdaie B, Loeb S, et al. An update of the American Urological Association White Paper on the prevention and treatment of the more common complications related to prostate biopsy. J Urol. 2017; 198:329.

5. Davis P, Paul E, Grummet J. Current practice of prostate biopsy in Australia and New Zealand: A survey. Urol Ann. 2015; 7:315-9.

6. Grummet J, Pepdjonovic L, Huang S, et al. Transperineal vs. transrectal biopsy in MRI targeting. Transl Androl Urol. 2017; 6:368.

7. Roberts MJ, Bennett HY, Harris PN, et al. Prostate Biopsy-related Infection: A Systematic Review of Risk Factors, Prevention Strategies, and Management Approaches. Urology. 2017; 104:11-21.

8. Pepdjonovic L, Tan GH, Huang S, et al. Zero hospital admissions for infection after 577 transperineal prostate biopsies using singledose cephazolin prophylaxis. World J Urol. 2017; 35:1199.

9. Murphy DG and Grummet JP. Planning for the post-antibiotic era - why we must avoid TRUS-guided biopsy sampling. Nat Rev Urol. 2016; 13:559-60.

10. D'Amico AV, Tempany CM, Cormack R, et al. Transperineal magnetic resonance image guided prostate biopsy. J Urol. 2000; 164:385-7.

11. Pepe P, Garufi A, Priolo GD, et al. Multiparametric MRI/TRUS Fusion Prostate Biopsy: Advantages of a Transperineal Approach. Anticancer Res. 2017; 37:3291-3294.

12. Hansen NL, Kesch C, Barrett T, et al. Multicentre evaluation of targeted and systematic biopsies using magnetic resonance and ultrasound image fusion guided transperineal prostate biopsy in patients with a previous negative biopsy. BJU Int. 2017; 120:631-8.

13. Radtke JP, Schwab C, Wolf MB, et al. Multiparametric magnetic resonance imaging (MRI) and MRI-transrectal ultrasound fusion biopsy for index tumor detection: correlation with radical prostatectomy specimen. Eur Urol. 2016; 70:846-53.

14. Tan N, Margolis DJ, Lu DY, et al. Characteristics of detected and missed prostate cancer foci on 3-T multiparametric MRI using an endorectal coil correlated with whole-mount thin-section histopathology. AJR Am J Roentgenol. 2015; 205:W87-92.

15. Le JD, Tan N, Shkolyar E, et al. Multifocality and prostate cancer detection by multiparametric magnetic resonance imaging: correlation with whole-mount histopathology. Eur Urol. 2015; 67:56976.

16. Seo JW, Shin SJ, Taik Oh Y, et al. PI-RADS version 2: detection of clinically significant cancer in patients with biopsy gleason score 6 prostate cancer. AJR Am J Roentgenol. 2017; 209:W1-9.

17. Matoso A, Epstein JI. Defining clinically significant prostate cancer on the basis of pathological findings. Histopathology. 2019; 74:135-145.

18. Heidenreich A, Bellmunt J, Bolla $M$, et al. EAU guidelines on prostate cancer. Part 1: screening, diagnosis, and treatment of clinically localised disease. Eur Urol. 2011; 59:61-71.

19. Thompson JE, Moses D, Shnier R, et al. Multiparametric magnetic resonance imaging guided diagnostic biopsy detects significant prostate cancer and could reduce unnecessary biopsies and over detection: a prospective study. J Urol. 2014; 192:67-74. 
20. Arumainayagam N, Ahmed HU, Moore CM, et al. Multiparametric MR imaging for detection of clinically significant prostate cancer: a validation cohort study with transperineal template prostate mapping as the reference standard. Radiology. 2013; 268:761-9.

21. Zhao C, Gao G, Fang D, et al. The efficiency of multiparametric magnetic resonance imaging (mpMRI) using PI-RADS version 2 in the diagnosis of clinically significant prostate cancer. Clin Imaging. 2016; 40:885-8.

22. Rosenkrantz AB, Verma S, Choyke P, et al. Prostate magnetic resonance imaging and magnetic resonance imaging targeted biopsy in patients with a prior negative biopsy: a consensus statement by AUA and SAR. J Urol. 2016; 196:1613-8.

23. Hakozaki $Y$, Matsushima $H$, Kumagai J, et al. A prospective study of magnetic resonance imaging and ultrasonography (MRI/US) fusion targeted biopsy and concurrent systematic transperineal biopsy with the average of 18-cores to detect clinically significant prostate cancer. BMC Urology. 2017; 17:117.

24. Valerio M, Donaldson I, Emberton M, et al. Detection of clinically significant prostate cancer using magnetic resonance imagingultrasound fusion targeted biopsy: a systematic review. Eur Urol. 2015; 68:8-19.

25. D'Agostino D, Mineo Bianchi F, Romagnoli D, et al. MRI/TRUS FUSION guided biopsy as first approach in ambulatory setting:
Feasibility and performance of a new fusion device. Arch Ital Urol Androl. 2020; 91:211-217.

26. Vyas L, Acher P, Kinsella J, et al. Indications, results and safety profile of transperineal sector biopsies (TPSB) of the prostate: a single centre experience of 634 cases. BJU Int. 2014; 114:32-37.

27. Loeb S, van den Heuvel S, Zhu X, et al. Infectious complications and hospital admissions after prostate biopsy in a European randomized trial. Eur Urol. 2012; 61:1110-4.

28. Chang DT, Challacombe B, Lawrentschuk N. Transperineal biopsy of the prostate-is this the future? Nat Rev Urol. 2013; 10:690-702.

29. Gaziev G, Wadhwa K, Barrett T, et al. Defining the learning curve for multiparametric magnetic resonance imaging (MRI) of the prostate using MRI-transrectal ultrasonography (TRUS) fusionguided transperineal prostate biopsies as a validation tool. BJU Int. 2016; 117:80-6.

30. Donato P, Morton A, Yaxley J, et al. Improved detection and reduced biopsies: the effect of a multiparametric magnetic resonance imaging-based triage prostate cancer pathway in a public teaching hospital. World J Urol. 2020; 38:371-379.

31. Pepe P, Aragona F. Morbidity after transperineal prostate biopsy in 3000 patients undergoing 12 vs 18 vs more than 24 needle cores. Urology. 2013; 81:1142-1146.

\section{Correspondence}

Shahbaz Mehmood, MD

Assistant Consultant Urologist

shahbazmalik49@gmail.com

Khalid Ibraheem Alothman, MD (Corresponding Author)

Consultant Urologist

kialothman@gmail.com

Abdulaziz Alwuhaibi, MD

Senior Resident Urology

abdulaziz.a.w@hotmail.com

Samia Mohamed Alhashim, MD

Biostatistician

samia@kfshrc.edu.sa

King Faisal Specialist Hospital \& Research Center Riyadh (KSA) 